\title{
Estratégias para o enfrentamento do absenteísmo de pacientes em consultas e exames agendados pelos sistemas de saúde: Uma revisão integrativa
}

Strategies for coping with patient absenteeism in consultations and examinations scheduled by health systems: An integrative review

Estrategias para afrontar el absentismo de los pacientes en las consultas y exámenes programados por los sistemas de salud: Una revisión integradora

\section{Resumo}

Objetivo: identificar os fatores que influenciam nas faltas de pacientes às consultas e exames agendados pelos sistemas de saúde, bem como analisar as principais estratégias adotadas para reduzir o absenteísmo. Métodos: trata-se de uma revisão integrativa com abordagem qualitativa, onde foi realizado um levantamento bibliográfico dos últimos anos nas bases de dados LILACS, MEDLINE e SciELO, bem como na biblioteca virtual Google Scholar. Resultados: Os artigos revelaram uma multicausalidade de fatores que geram absenteísmo, os quais pode-se citar o tempo de espera, esquecimento, falhas na comunicação, e barreiras socioculturais e econômicas. As principais medidas estratégicas incluem melhoria do acesso aos usuários, redução das filas de espera, comunicação eficiente, e fortalecimento da Regulação assistencial. Conclusão: acredita-se que a redução nas perdas por absenteísmo de pacientes deve partir de abordagens mistas, de acordo com as necessidades da população envolvida, facilitando cancelamentos sempre que necessário, observando a qualidade na comunicação, considerando a integração entre atenção básica e especializada.

Palavras-chave: Atenção à saúde; Agendamento de consultas; Acesso aos serviços de saúde; Absenteísmo; Revisão sistemática. 


\begin{abstract}
Objective: to identify the factors that influence patients' absences from appointments and exams scheduled by health systems, as well as to analyze the main strategies adopted to reduce absenteeism. Methods: it is an integrative review with a qualitative approach, being based on a literature of the last years was carried out in the LILACS, MEDLINE and SciELO databases, as well as in the Google Scholar virtual library. Results: the articles revealed a multiple causality of factors that generate absenteeism, which can be mentioned: waiting time, forgetfulness, communication failures, and socio-cultural and economic barriers. The main strategic measures include improving access to users, reducing queues, efficient communication, and strengthening assistance regulation. Conclusion: it is believed that the reduction in losses due to patient absenteeism should start from mixed approaches, according to the needs of the population involved, facilitating cancellations whenever necessary, observing the quality of communication, considering the integration between basic and specialized care.
\end{abstract}

Keywords: Universal access to health care services; Appointments and schedules; Health services accessibility; Absenteeism; Systematic review.

\title{
Resumen
}

Objetivo: identificar los motivos de las ausencias de los pacientes a las citas y exámenes programados, así como analizar las principales estrategias adoptadas para reducir el absentismo. Métodos: se trata de una revisión integradora con enfoque cualitativo, donde se realizó un levantamiento bibliográfico de los últimos años en las bases de datos LILACS, MEDLINE y SciELO, así como en la biblioteca virtual Google Scholar. Resultados: Los artículos revelaron una multicausalidad de factores generadores de absentismo, entre los que se pueden mencionar: tiempo de espera, olvidos, fallas de comunicación y barreras socioculturales y económicas. Las principales medidas estratégicas incluyen mejorar el acceso a los usuarios, reducir las colas, una comunicación eficiente y fortalecer la regulación de la asistencia. Conclusión: la reducción del absentismo de los pacientes debe partir de abordajes mixtos, de acuerdo con las necesidades de la población involucrada, facilitando cancelaciones cuando sea necesario, observando la calidad de la comunicación, considerando la integración entre atención básica y especializada.

Palabras clave: Acceso universal a los servicios de salud; Citas y horarios; Accesibilidad a los servicios de salud; Absentismo; Revisión sistemática.

\section{Introdução}

No âmbito do Sistema Único de Saúde (SUS), tem-se as Unidades Básicas de Saúde, onde é oferecida a atenção primária, seguindo pelas unidades de atenção média, como ambulatórios especializados e, então, chegando a alta complexidade em centros de atenção terciária e quaternária, onde são realizados procedimentos mais complexos, como o transplante de órgãos, garantindo assim o acesso integral e universal, de forma gratuita, para mais de 180 milhões de pessoas no Brasil (Souza, 2002).

Por outro lado, esse sistema enfrenta diversos desafios ao longo da sua existência ainda recente, sendo um deles referente ao agendamento de consultas e exames, onde o absenteísmo de pacientes nesses procedimentos reflete dificuldades que vão de encontro ao que é preconizado como acesso integral e universal dos usuários.

O absenteísmo de pacientes em consultas agendadas configura um grande problema para as organizações, provocando subutilização dos recursos empregados, gerando desperdícios financeiros, interferindo na eficiência dos sistemas de saúde e, consequentemente, comprometendo a saúde dos usuários em virtude do esforço extra para reagendar os procedimentos (Fenili et al., 2017; Hasvold \& Wootton, 2011; Zirkle \& McNelles, 2011; Gurol-Urganci et al, 2002). Somado a isso, observa-se uma circulação exaustiva em rede por parte do usuário e novas filas de espera, o que contribui ainda mais para causas que levam ao absenteísmo, como, por exemplo, esquecer o agendamento (Cavalcanti et al., 2013).

A ausência de articulação entre os níveis de atenção e o desconhecimento dos profissionais de saúde que oriente a ordenação das solicitações de encaminhamento às consultas e exames agendados contribui significativamente com a elevação do número de solicitações de procedimentos sem critérios, gerando demanda reprimida, bem como causando desmotivação da equipe de saúde, insatisfação/descrédito do usuário no sistema, levando, inclusive, ao agravamento da sua doença.

Tais fatos apresentam-se diante da necessidade de se repensar o papel da Atenção Primária a Saúde (APS) como ordenadora do processo de cuidado, bem como da capacidade integradora de se trabalhar em redes de saúde de modo que esses problemas possam ser minimizados ou até sanados. 
Nesse contexto, o absenteísmo tem se revelado como um problema crônico na rede pública de saúde brasileira, onde a ausência dos usuários nas consultas e exames agendados atinge altos índices por parte dos usuários, o que implica em uma baixa utilização de recursos, fato que pode ser percebido em todas as regiões do Brasil nos mais diversos tipos de atendimentos e especialidades (Oleskovicz et al., 2020).

Os motivos para o absenteísmo de pacientes em consultas e exames especializados são variados e culminam em perdas de recursos públicos. Além disso, prejuízos na continuidade da assistência e na resolutividade das demandas de saúde também são impactantes, pois contribuem para o aumento das filas de espera e das demandas por urgência em consequência dessas faltas (Jandrey \& Drehmer, 2000).

Diante do exposto, esta pesquisa objetiva responder as seguintes perguntas: quais os motivos que levam os pacientes a faltar às consultas e exames agendados? Que estratégias podem ser tomadas para enfrentar as consequências dessa problemática para o sistema de saúde em geral? Portanto, a partir de uma revisão integrativa, com base em levantamentos bibliográficos entre 2015 e 2020, este estudo visa contribuir com o novel de investigações sobre o absenteísmo de pacientes na assistência à saúde.

\section{Metodologia}

Segundo Fonseca (2002, p. 32), "qualquer trabalho científico começa com uma pesquisa bibliográfica, o que permite ao pesquisador saber o que já foi estudado sobre o assunto". Assim, a essência deste estudo envolve o levantamento bibliográfico, o qual tem a finalidade de sintetizar de maneira sistemática, ordenada e abrangente, os resultados provenientes da pesquisa bibliográfica acerca do tema abordado.

Portanto, esta pesquisa se trata de uma revisão integrativa de literatura sobre os aspectos que envolvem as causas e as estratégias de enfrentamento do absenteísmo de pacientes em consultas e exames agendados. Nesse sentido, tem-se que a revisão integrativa "oferece aos profissionais de diversas áreas de atuação na saúde o acesso rápido aos resultados relevantes de pesquisas que fundamentam as condutas ou a tomada de decisão, proporcionando um saber crítico" (Mendes et al., 2008, p. 765).

Uma vez que o foco desta pesquisa não consiste na quantificação dos resultados, utilizou-se a abordagem qualitativa para a análise e interpretação dos dados, que permitirá o embasamento para discussões mais amplas sobre o assunto abordado. Diante de sua natureza complexa, Minayo (1994, p. 21-22), observa que:

A pesquisa qualitativa responde a questões muito particulares. Ela se preocupa, nas ciências sociais, com um nível de realidade que não pode ser qualificado, ou seja, ela trabalha com o universo de significados, motivos, aspirações, crenças, valores e atitudes, o que corresponde a um espaço mais profundo das relações, dos processos e dos fenômenos que não podem ser reduzidos a operacionalização de variáveis. (Minayo, 1994, p. 21-22)

A busca dos estudos ocorreu nas bases de dados LILACS, MEDLINE e SciELO, bem como no buscador acadêmico Google Scholar (Google Acadêmico). Foram utilizadas combinações entre as seguintes palavras-chave, consideradas descritores no DeCS (Descritores em Ciências de Saúde): Regulação e Fiscalização em Saúde (Health Care Coordination and Monitoring); Absenteísmo (Absenteeism); Agendamento de Consultas; Especialidade Médica (Medicine); Exames Médicos (Medical Examination); Atenção Primária à Saúde (Primary Health Care); Acesso aos Serviços de Saúde (Health Services Accessibility). Os termos foram cruzados como descritores e também como palavras do título e do resumo, assim, foram selecionados os artigos disponibilizados na íntegra (tanto em português, inglês e/ou espanhol), sendo considerado o período de 2015 a 2020, uma vez que se intenciona investigar as produções científicas mais recentes sobre o tema.

Com a seleção dos artigos a partir do cruzamento entre os descritores, os trabalhos tiveram seus resumos lidos e, 
seguindo os critérios de inclusão, aqueles que estavam em desacordo com o objetivo desta pesquisa foram então excluídos. Dessa maneira, foram selecionados os artigos considerados com potenciais para a leitura na íntegra e então foi verificado quais responderam às perguntas norteadoras desta pesquisa para, assim, serem incluídos na revisão integrativa.

A análise dos resultados se deu de forma descritiva, observando as potenciais causas do absenteísmo em consultas e exames encontrados. As estratégias de enfrentamento foram sistematizadas separadamente.

\section{Resultados}

A amostra desta pesquisa foi constituída por 126 artigos científicos encontrados nas bases de dados, selecionados para leitura analítica partir de critérios de inclusão previamente estabelecidos. Desse modo, 28 foram selecionados como objeto de estudo para serem lidos na íntegra, dentre os quais 20 à pergunta norteadora desta pesquisa (Figura 1).

Figura 1 - Fluxograma do processo de busca, seleção e inclusão das publicações para a revisão integrativa.

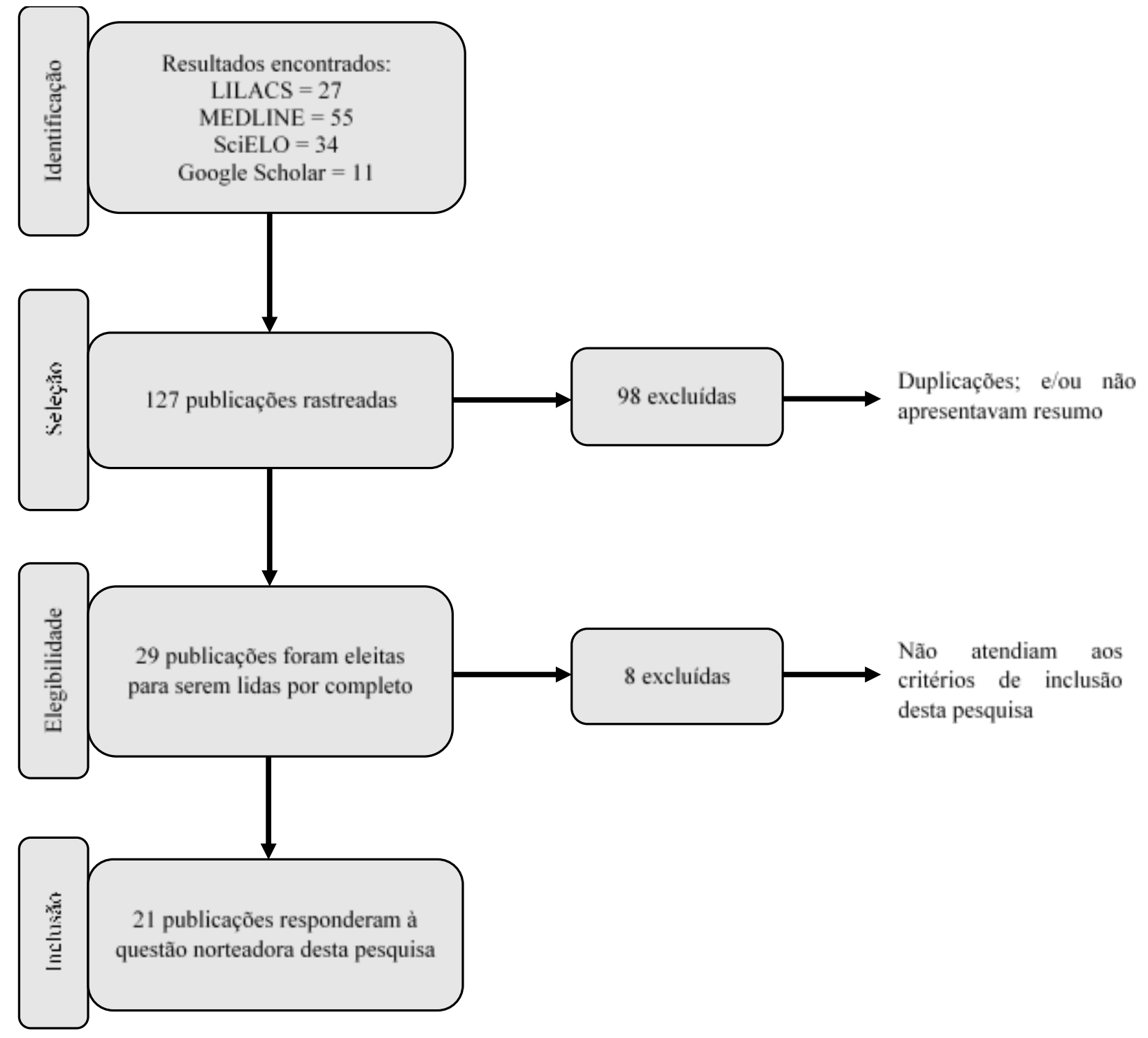

Fonte: Autores (2021).

Na Tabela 1 são apresentados os estudos selecionados para esta revisão integrativa, com seus respectivos autores e ano de publicação, títulos, local de busca, tipo de pesquisa e suas principais conclusões. 


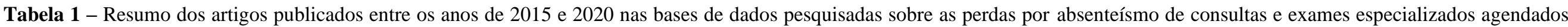
pelas centrais de regulação dos municípios brasileiros.

\begin{tabular}{|c|c|c|c|c|}
\hline $\begin{array}{l}\text { Autor(es) e ano de } \\
\text { publicação }\end{array}$ & Título da pesquisa & Local de busca & Tipo de pesquisa & Principais conclusões \\
\hline Farias et al. (2020) & $\begin{array}{l}\text { Absenteísmo de usuários: barreiras e } \\
\text { determinantes no acesso aos serviços } \\
\text { de saúde }\end{array}$ & LILACS & $\begin{array}{l}\text { Metassíntese como } \\
\text { modalidade de revisão de } \\
\text { literatura }\end{array}$ & $\begin{array}{l}\text { O conhecimento das barreiras e determinantes do acesso permite } \\
\text { compreender as possíveis causas do absenteísmo e suas consequências, a fim } \\
\text { de fundamentar a tomada de decisões que possibilitem a correção ou } \\
\text { minimização de riscos e de prejuízos econômicos, na administração dos } \\
\text { serviços públicos de saúde. }\end{array}$ \\
\hline Rodrigues et al. (2020) & $\begin{array}{l}\text { Impacto das mensagens de texto para } \\
\text { redução do absenteísmo às consultas } \\
\text { especializadas: um estudo aleatorizado }\end{array}$ & LILACS & $\begin{array}{l}\text { Estudo do tipo experimental } \\
\text { de natureza longitudinal }\end{array}$ & $\begin{array}{l}\text { Embora se saiba que o absenteísmo é multicausal e envolve o usuário, o } \\
\text { prestador de serviço de saúde e a gestão do sistema, a razão mais citada na } \\
\text { literatura como causa de absenteísmo às consultas é o esquecimento do } \\
\text { compromisso. Outras situações como necessidade de transporte até o serviço } \\
\text { de saúde, fatores socioeconômicos, custeios para alimentação, obrigações } \\
\text { trabalhistas e escolares, dependência de um familiar ou acompanhante } \\
\text { contribuem para o absenteísmo às consultas eletivas agendadas. }\end{array}$ \\
\hline Briatore et al. (2020) & $\begin{array}{l}\text { Causes of appointment attendance, } \\
\text { nonattendance, and cancellation in } \\
\text { outpatient consultations at a university } \\
\text { hospital }\end{array}$ & MEDLINE & $\begin{array}{l}\text { Estudo de caso e dois } \\
\text { controles aninhados em uma } \\
\text { coorte prospectiva }\end{array}$ & $\begin{array}{l}\text { A principal causa do não comparecimento é o esquecimento da consulta } \\
\text { agendada, mas há uma proporção de diferentes causas que não respondem } \\
\text { aos lembretes, mas podem responder a diferentes estratégias }\end{array}$ \\
\hline Beltrame et al. (2019) & $\begin{array}{l}\text { Absenteísmo de usuários como fator de } \\
\text { desperdício: desafio para } \\
\text { sustentabilidade em sistema universal } \\
\text { de saúde }\end{array}$ & SciELO & Estudo descritivo & $\begin{array}{l}\text { Os valores totais desperdiçados são significativos e evidenciam o desafio } \\
\text { constante na agenda dos gestores na busca pela SUStentabilidade em } \\
\text { sistemas universais de saúde. }\end{array}$ \\
\hline
\end{tabular}


Research, Society and Development, v. 10, n. 7, e46210716671, 2021

(CC BY 4.0) | ISSN 2525-3409 | DOI: http://dx.doi.org/10.33448/rsd-v10i7.16671

\begin{tabular}{|c|c|c|c|c|}
\hline Mazza et al. (2019) & $\begin{array}{l}\text { Fatores do absenteísmo em primeira } \\
\text { consulta num ambulatório de oncologia } \\
\text { em um Hospital Universitário }\end{array}$ & LILACS & Pesquisa de campo & $\begin{array}{l}\text { As principais razões para o absenteísmo estão relacionadas ao indivíduo e } \\
\text { que problemas de cadastro do paciente e comunicação dificultam a } \\
\text { compreensão das causas do absenteísmo. }\end{array}$ \\
\hline Campoville (2019) & $\begin{array}{l}\text { Estudo sobre absenteísmo em } \\
\text { ambulatórios médicos especializados } \\
\text { no interior de São Paulo }\end{array}$ & Google Scholar & Quanti-qualitativa & $\begin{array}{l}\text { Foram encontrados } 3 \text { principais motivos do absenteísmo a partir da } \\
\text { percepção dos gestores à saber: responsabilização do paciente, melhora do } \\
\text { quadro de saúde e tempo na fila de espera. [...] }\end{array}$ \\
\hline Farias et al. (2019) & $\begin{array}{l}\text { Tempo de espera e absenteísmo na } \\
\text { atenção especializada: um desafio para } \\
\text { os sistemas universais de saúde }\end{array}$ & LILACS & $\begin{array}{l}\text { Estudo descritivo } \\
\text { retrospectivo }\end{array}$ & $\begin{array}{l}\text { O estudo demonstrou que existe uma correlação positiva entre o tempo de } \\
\text { espera e o absenteísmo e o porte municipal. De modo geral, o tempo de } \\
\text { espera, que envolve o processo regulatório de agendamento das consultas e } \\
\text { exames especializados é fator importante que demonstrou influenciar o } \\
\text { absenteísmo. Além disso, o tempo de espera entre a solicitação e o } \\
\text { agendamento e a maior disponibilidade tecnológica em saúde nos municípios } \\
\text { de grande porte parecem fazer com que o absenteísmo seja maior nesses } \\
\text { municípios. }\end{array}$ \\
\hline Pires Filho et al. (2019) & $\begin{array}{l}\text { Acesso Avançado em uma Unidade de } \\
\text { Saúde da Família do interior do estado } \\
\text { de São Paulo: um relato de experiência }\end{array}$ & LILACS & Estudo de caso & $\begin{array}{l}\text { A equipe, em especial os funcionários da recepção da unidade, consideraram } \\
\text { que a redução do tempo de espera facilitou o fluxo de trabalho diário. Toda a } \\
\text { equipe considerou que a redução do absenteísmo foi benéfica à } \\
\text { longitudinalidade do cuidado esperada na ESF. }\end{array}$ \\
\hline Silveira et al. (2018) & $\begin{array}{l}\text { Prevalência de absenteísmo em } \\
\text { consultas médicas em unidade básica } \\
\text { de saúde do sul do Brasil }\end{array}$ & LILACS & Estudo transversal & $\begin{array}{l}\text { Os resultados mostram uma elevada prevalência de absenteísmo, } \\
\text { especialmente nas consultas de clínica médica, o que pode acarretar } \\
\text { problemas tanto para a continuidade do cuidado, especialmente aos usuários } \\
\text { da clínica geral, quanto para o ensino médico na APS. }\end{array}$ \\
\hline Costa et al. (2018) & $\begin{array}{l}\text { Absenteísmo em consultas } \\
\text { especializadas referenciadas por } \\
\text { unidade básica saúde da família: estudo }\end{array}$ & Google Scholar & Estudo de caso & $\begin{array}{l}\text { Documentos a serem encaminhados para a Central de Marcação exigem } \\
\text { atenção criteriosa. O acompanhamento dos Agentes Comunitários de Saúde } \\
\text { aos usuários antes e pós-consulta é uma ação para diminuir o absenteísmo. A }\end{array}$ \\
\hline
\end{tabular}


Research, Society and Development, v. 10, n. 7, e46210716671, 2021

(CC BY 4.0) | ISSN 2525-3409 | DOI: http://dx.doi.org/10.33448/rsd-v10i7.16671

\begin{tabular}{|c|c|c|c|c|}
\hline & de caso & & & solução exige conscientização dos usuários. \\
\hline Cruz et al. (2018) & $\begin{array}{l}\text { A regulação da atenção à saúde bucal e } \\
\text { o absenteísmo: cenários e } \\
\text { possibilidades }\end{array}$ & Google Scholar & $\begin{array}{l}\text { Revisão integrativa da } \\
\text { literatura }\end{array}$ & $\begin{array}{l}\text { Se por um lado há uma queixa de elevada quantidade de exames e consultas, } \\
\text { por outro há um desperdício significativo de ofertas, o que interfere na } \\
\text { qualidade da atenção à saúde. }\end{array}$ \\
\hline Siqueira (2018) & $\begin{array}{l}\text { Análise do impacto da fila de espera na } \\
\text { probabilidade de absenteísmo em } \\
\text { exames e consultas }\end{array}$ & Google Scholar & Qualitativo & $\begin{array}{l}\text { Ficou evidente que a redução do tempo de espera para menos de } 60 \text { dias } \\
\text { possibilitará a diminuição significativa do absenteísmo, o que repercute na } \\
\text { diminuição do agravamento de doenças e nos custos ao sistema de saúde. }\end{array}$ \\
\hline Cavalcanti et al. (2018) & $\begin{array}{l}\text { Desafios da Regulação Assistencial na } \\
\text { Organização do Sistema Único de } \\
\text { Saúde }\end{array}$ & Google Scholar & Bibliográfica & $\begin{array}{l}\text { A precariedade de sistemas de informação e comunicação apresentou } \\
\text { fragilidades no gerenciamento dos referenciamentos, de demanda reprimida e } \\
\text { do absenteísmo de consultas especializadas e exames, tais como a ausência de } \\
\text { controles ou controles fragmentados e inconsistentes. [...] Parte desse } \\
\text { absenteísmo e a baixa cobertura da atenção básica podem estar sendo } \\
\text { responsáveis pelo acesso direto do usuário aos serviços especializados sem } \\
\text { passar pela porta de entrada, o que contribuiria para a não diminuição da fila de } \\
\text { espera. }\end{array}$ \\
\hline $\begin{array}{l}\text { Hernández García et al. } \\
\text { (2017) }\end{array}$ & $\begin{array}{l}\text { Absentismo, y factores asociados, en } \\
\text { las citas programadas de una consulta } \\
\text { externa de Medicina Preventiva }\end{array}$ & MEDLINE & Estudo transversal & $\begin{array}{l}\text { A detecção de certos fatores associados (idade, país de nascimento, hora da } \\
\text { consulta, motivo da consulta - vacinação de pacientes com insuficiência renal } \\
\text { crônica -- e dia da semana) tornou possível planejar medidas de melhoria com a } \\
\text { necessidade de modificar a situação de absenteísmo. }\end{array}$ \\
\hline Miranda (2017) & $\begin{array}{l}\text { A implantação de aplicativo para } \\
\text { acompanhamento de consultas e } \\
\text { exames como estratégia para diminuir } \\
\text { o absenteísmo dos usuários no } \\
\text { município de Caruaru- PE }\end{array}$ & LILACS & Intervenção & $\begin{array}{l}\text { A Secretaria Executiva de Regulação do município adotou ações para } \\
\text { acompanhamento do absenteísmo e através de contato telefônico com os } \\
\text { usuários faltosos descobriu que o fenômeno é multicausal, sendo um dos } \\
\text { motivos de ausência, a falta de comunicação com o usuário. }\end{array}$ \\
\hline
\end{tabular}


Research, Society and Development, v. 10, n. 7, e46210716671, 2021

(CC BY 4.0) | ISSN 2525-3409 | DOI: http://dx.doi.org/10.33448/rsd-v10i7.16671

\begin{tabular}{|c|c|c|c|c|}
\hline Bittar et al. (2016) & $\begin{array}{l}\text { Absenteísmo em atendimento } \\
\text { ambulatorial de especialidades no } \\
\text { estado de São Paulo }\end{array}$ & Google Scholar & Descritiva & $\begin{array}{l}\text { Entre as causas encontram-se aquelas ligadas a fatores socioeconômicos e } \\
\text { comportamentais, exigindo que diferentes categorias profissionais trabalhem } \\
\text { em equipe, além de propostas estruturadas e uso intensivo de tecnologia de } \\
\text { comunicação. }\end{array}$ \\
\hline Foltz (2016) & $\begin{array}{l}\text { Enfrentamento do absenteísmo no } \\
\text { centro de especialidades médicas e } \\
\text { odontológicas de Araucária/PR }\end{array}$ & Google Scholar & Quanti-qualitativa & $\begin{array}{l}\text { As ações necessárias para a efetiva redução das faltas dos pacientes envolvem } \\
\text { medidas complexas e variadas, nos níveis estratégico, tático e operacional, com } \\
\text { a implantação de estratégias focais e sistêmicas, e de sistemas de avaliação e } \\
\text { monitoramento. }\end{array}$ \\
\hline Rocha (2015) & $\begin{array}{l}\text { Regulação assistencial ambulatorial no } \\
\text { Município do Rio de Janeiro, RJ: } \\
\text { efeitos da inserção da APS na } \\
\text { regulação }\end{array}$ & LILACS & Análise comparativa & $\begin{array}{l}\text { A redução dos tempos de espera resultaram de um conjunto diversificado de } \\
\text { medidas tomadas pela SMS do Rio de Janeiro. As mudanças na regulação } \\
\text { melhoraram sua qualidade e foram mais efetivas para redução de tempos de } \\
\text { espera quando acompanhadas do aumento da oferta de vagas. }\end{array}$ \\
\hline Torezani (2015) & $\begin{array}{l}\text { Estudo sobre as faltas às consultas e } \\
\text { seus motivos: uma análise da } \\
\text { comunidade Sete Anões - Mesquita/RJ }\end{array}$ & Google Scholar & Qualitativo & $\begin{array}{l}\text { Dentre os problemas encontrados estão diferenças culturais e hábitos locais, } \\
\text { treinamento escasso de agentes comunitários de saúde e falhas no sistema de } \\
\text { marcações. }\end{array}$ \\
\hline Jabalera Mesa et al. (2015) & $\begin{array}{l}\text { Factores determinantes y coste } \\
\text { económico del absentismo de pacientes } \\
\text { en consultas externas de la Agencia } \\
\text { Sanitaria Costa del Sol }\end{array}$ & MEDLINE & $\begin{array}{l}\text { Estudo observacional, } \\
\text { multicêntrico de casos e } \\
\text { controles }\end{array}$ & $\begin{array}{l}\text { As principais razões para o absenteísmo são evitáveis e intervenções como } \\
\text { procedimentos de comunicação aprimorados ou sistemas de lembretes de } \\
\text { compromissos podem ser benéficos. }\end{array}$ \\
\hline Albieri et al. (2015) & $\begin{array}{l}\text { Agilidade no acesso do cidadão a partir } \\
\text { da gestão de fila de espera da atenção } \\
\text { especializada ambulatorial: em busca } \\
\text { da melhoria da eficácia da rede de } \\
\text { atenção à saúde }\end{array}$ & Google Scholar & Pesquisa-ação & $\begin{array}{l}\text { A soma de diferentes ações como implementação de ferramentas de gestão, } \\
\text { contratualização com prestadores, aperfeiçoamento de processos de trabalho e } \\
\text { renovação das estratégias nos vale de resultados positivos diante da ampliação } \\
\text { da oferta de serviços e redução da fila de espera. }\end{array}$ \\
\hline
\end{tabular}

Fonte: Autores (2021). 
As principais causas de absenteísmo de consultas e exames especializados agendados, de acordo com a leitura dos artigos selecionados para esta revisão integrativa, são as barreiras socioculturais (20,63\%), tempo de espera e problemas de comunicação $(15,87 \%)$, respectivamente (Tabela 2$)$.

Tabela 2 - Frequência das principais causas de absenteísmo de pacientes em consultas e exames agendados de acordo com os estudos da revisão integrativa entre 2015 e 2020.

\begin{tabular}{l|c}
\hline \multicolumn{1}{c|}{ Causas de absenteísmo de pacientes em consultas e exames agendados } & Frequência (\%) \\
\hline $\begin{array}{l}\text { Barreiras socioculturais (obrigações trabalhistas e/ou escolares, diferenças } \\
\text { culturais e hábitos locais, dia/horário da semana do agendamento) }\end{array}$ & $20,63 \%$ \\
\hline Tempo de espera & $15,87 \%$ \\
\hline Problemas de comunicação & $15,87 \%$ \\
\hline Esquecimento & $11,11 \%$ \\
\hline Barreiras econômicas (custeio para alimentação e/ou transporte até o local & $11,11 \%$ \\
\hline da consulta/exame) & $4,76 \%$ \\
\hline Melhora do quadro de saúde & $4,76 \%$ \\
\hline Dependência de um familiar & $4,76 \%$ \\
\hline Organização da agenda e disponibilidade de vagas para consultas e exames & $4,76 \%$ \\
\hline Escassez de recursos tecnológicos nos estabelecimentos de saúde & $3,17 \%$ \\
\hline Problemas técnico funcionais e/ou falhas no sistema de marcações & $1,59 \%$ \\
\hline Treinamento escasso do agente comunitário de saúde (ACS) quanto ao & $1,59 \%$ \\
\hline acompanhamento e lembrete dos agendamentos aos usuários & \\
\hline Influência política na gestão nos estabelecimentos de saúde & \\
\hline
\end{tabular}

Fonte: Autores (2021).

Diante disso, tem-se que a maioria das causas do não comparecimento de usuários em consultas e exames agendados pode ser evitada (Farias et al., 2020; Rocha, 2015; Jabalera Mesa \& Asencio, 2015). Estratégias para o enfrentamento do absenteísmo devem, inicialmente, buscar manter os dados dos pacientes atualizados e dispor de maneiras efetivas de compartilhamento de informações com a rede de atenção à saúde, uma vez que problemas cadastrais e de comunicação constituem dificuldades para a compreender o que causam as das faltas, bem como o pleno desenvolvimento de ações de combate a esse problema (Mazza et al., 2019).

É importante ter conhecimento das características e distribuição das faltas, visto que uma possível redução do absenteísmo de usuários pode implicar na passagem de faltas para cancelamentos (Briatore et al., 2019). Assim, as estratégias definidas devem considerar, além do sistema de saúde em si, os diversos atores envolvidos na atenção à saúde.

Os resultados desta pesquisa revelaram diversas estratégias que podem ser adotadas em razão do absenteísmo de pacientes em consultas e exames agendados, onde se destacam melhorias na comunicação e lembretes dos usuários quanto aos agendamentos (21,05\%), bem como o controle da fila de espera (13,16\%) (Tabela 3$)$. 
Tabela 3 - Estratégias propostas para o enfrentamento do absenteísmo de usuários em consultas e exames agendados pelos sistemas de saúde de acordo com os estudos da revisão integrativa entre 2015 e 2020.

\begin{tabular}{|c|c|}
\hline $\begin{array}{l}\text { Estratégias para diminuir o absenteísmo de pacientes citadas nos estudos } \\
\text { incluídos na revisão integrativa }\end{array}$ & Frequência $(\%)$ \\
\hline Melhorar a comunicação com os usuários e efetivar mecanismos de lembretes & $21,05 \%$ \\
\hline $\begin{array}{l}\text { Reduzir/qualificar a fila de espera, além de promover o controle da fila através da } \\
\text { abertura das agendas com antecedência }\end{array}$ & $13,16 \%$ \\
\hline $\begin{array}{l}\text { Melhorar o acesso dos usuários, o que requer investimentos e esforços para } \\
\text { identificar, sob a ótica dos usuários e profissionais, áreas prioritárias de atuação }\end{array}$ & $10,53 \%$ \\
\hline Overbooking (agendamento maior de pacientes, além da capacidade instalada) & $7,89 \%$ \\
\hline Definição/reestruturação de fluxos e protocolos & $7,89 \%$ \\
\hline $\begin{array}{l}\text { Ampliação da oferta de consultas/exames, bem como monitoramento dos indicadores } \\
\text { e reprogramação permanente de ofertas com base nas necessidades de saúde das } \\
\text { regiões }\end{array}$ & $7,89 \%$ \\
\hline $\begin{array}{l}\text { Maior frequência de visitas dos ACSs, visando o acompanhamento desse } \\
\text { profissional antes e pós-consulta }\end{array}$ & $5,26 \%$ \\
\hline Criação de central de confirmação de agendamento (call center) & $5,26 \%$ \\
\hline Evitar agendamentos coincidentes em horários e datas; agendamento automático & $5,26 \%$ \\
\hline Melhor acompanhamento pelas equipes da Atenção Básica & $2,63 \%$ \\
\hline $\begin{array}{l}\text { Implementação do método Acesso Avançado para organização de agenda em } \\
\text { unidades de saúde na Atenção Primária à Saúde que prega a máxima "Faça hoje o } \\
\text { trabalho de hoje!" }\end{array}$ & $2,63 \%$ \\
\hline Uso de telessaúde, para qualificar o atendimento & $2,63 \%$ \\
\hline Sistemas integrados a Atenção Básica e Central de Regulação & $2,63 \%$ \\
\hline Empoderamento do usuário & $2,63 \%$ \\
\hline $\begin{array}{l}\text { Penalizações como o impedimento de remarcações automáticas dos pacientes } \\
\text { faltosos, ocorrendo o agendamento somente em caso de vaga de encaixe, melhora da } \\
\text { ambiência dos equipamentos de saúde, capacitação de equipes de saúde, e educação } \\
\text { em saúde }\end{array}$ & $2,63 \%$ \\
\hline
\end{tabular}

Fonte: Autores (2021).

Vale ressaltar que a adoção de certas estratégias é importante para evitar mais de uma causa de absenteísmo de pacientes, por exemplo, a melhoria na comunicação possibilita evitar o esquecimento do agendamento, o que pouparia também gastos por ociosidade de recursos devido o não comparecimento aos procedimentos e, por fim, evitando reagendamentos, ou seja, fazendo com que o paciente não precise entrar novamente na fila de espera, prolongando ainda mais o seu tratamento.

\section{Discussão}

Os trabalhos selecionados para esta revisão integrativa apontam perdas de consultas e exames por absenteísmos sob diversas perspectivas. Contudo, alguns autores são enfáticos ao ressaltar que o absenteísmo é uma fonte de desperdícios de 
recursos financeiros, materiais e humanos (Farias et al., 2020, Beltrame et al., 2019; Siqueira, 2018; Miranda, 2017; Bittar et al., 2016).

De modo geral, o absenteísmo em consultas e exames especializados é multicausal. No entanto, em algumas especialidades as causas podem ser mais específicas. Beltrame et al. (2019) mostraram que a baixa taxa de absenteísmo nas consultas e exames podem ser justificadas pelo agravamento do quadro patológico já diagnosticado, bem como pela necessidade de rápida intervenção, como foi observado no caso especialidade médica cancerologista cirúrgico, cuja taxa de absenteísmo foi de apenas $26,4 \%$. Em contrapartida, a consulta de fisioterapeuta geral foi a que deteve a maior quantidade de faltas $(75,8 \%)$ e, de acordo com a gerência da Central de Regulação, o absenteísmo se justifica por erros no registro de comparecimento, por limitações de locomoção em decorrência dos agravos da própria patologia e também, em alguns casos, pela melhoria nos sintomas.

O tempo de espera, bastante abordado nos estudos selecionados, desponta como um dos principais problemas envolvendo as perdas por absenteísmo de pacientes. De acordo com Siqueira (2018), obtém-se maiores probabilidades de absenteísmos em procedimentos que ultrapassam os 60 dias de espera. Nesse sentido, ao adotar medidas como o controle da fila de espera, conforme foi observado no trabalho de Bittar et al. (2016), através da abertura das agendas com antecedência de 2 meses, deixar o AME (Ambulatórios Médico de Especialidades) com o retorno no agendado e ligação para relembrar o paciente do procedimento, foram capazes de reduzir o número de pacientes em fila de espera de 2.815 (abril de 2014 ) para 313 (setembro de 2014) e o tempo de retorno passou de 120 dias para 40 dias.

Um dos estudos selecionados para a revisão integrativa versa sobre a implementação do método organizacional de agenda Acesso Avançado, elaborado por Murray e Tantau (2000). Também conhecido como "agendamento no mesmo dia", permite aos pacientes buscarem e receberem cuidados primários de saúde de seu profissional de referência, no momento de sua necessidade, geralmente no mesmo dia. Tendo como máxima "Faça hoje o trabalho de hoje!", a agenda então se permanece aberta e, conforme a demanda, seus horários são preenchidos diariamente.

Assim, a pesquisa realizada por Pires Filho et al. (2019) apresentou os resultados da implementação do Acesso Avançado, sendo observado uma diminuição do tempo médio de espera entre a procura do paciente à unidade de saúde e sua efetiva consulta, além de ter reduzido também o absenteísmo. Importante ressaltar que, embora os resultados sejam animadores, faz-se necessário investigar primeiramente o perfil populacional a fim de saber a respeito da aceitação quanto a introdução desse método.

Diminuir as filas de espera é importante, inclusive, para se combater outra causa de absenteísmo de pacientes, que é o esquecimento. Nesse sentido, quanto menor for o tempo esperando em fila, menor serão as chances de o usuário esquecer o agendamento. Estudos reforçaram ainda que estabelecer uma relação próxima entre o paciente e a unidade de saúde contribui para a redução no absenteísmo, de modo que acompanhamento do paciente de forma sistemática, verificando sua frequência a unidade, confirmando previamente os seus agendamentos e procedendo com adaptações nas confirmações não realizadas possibilitam reduzir a fila de espera (Albieri et al., 2015).

Essa proximidade deve ser ainda mais fortalecida ao se observar como outros fatores, mais subjetivos, tal como diferenças culturais e hábitos locais podem associar-se às faltas de pacientes. Logo, alguns autores observam a necessidade de que o ACS seja bastante presente no acompanhamento dos usuários, aumentando suas visitas, relembrando os pacientes dos procedimentos agendados, contribuindo para diminuir o esquecimento e, consequentemente, as taxas de absenteísmo. ${ }^{18,25}$ Esse fato contribui, sobretudo, para um melhor acompanhamento pelas equipes da Atenção Básica, o que também fortalece a redução das faltas. 
Obrigações trabalhistas e/ou escolares, dia/horário da semana do agendamento também incidem sobre as taxas de absenteísmo de pacientes. Desse modo, Hernández García (2018) chamam a atenção para a associação entre o dia da semana e as faltas, logo, intervenções para reduzir o absenteísmo devem observar essa relação.

Nitidamente, uma das principais estratégias utilizadas para reduzir as perdas geradas pelo absenteísmo é o reforço na comunicação, pois permite relembrar o agendamento e, assim, evitar o esquecimento do compromisso. Ligações e envio de emails ou mensagens de texto evidentemente são bastante eficazes para garantir a comunicação com o paciente, embora Rodrigues et al. (2020) tenha observado que o lembrete por mensagens de texto via internet, realizada através do aplicativo WhatsApp, não contribuiu significativamente para uma redução do absenteísmo.

Jabalera Mesa (2015) cita a disponibilização de um número de contato direto para o cancelamento de procedimentos, que está impresso na nota de consultas do paciente. Nesse contexto, alguns autores elencam a criação de call centers como forma de garantir essa comunicação entre o serviço e os usuários (Foltz, 2016; Albieri et al., 2015).

Outra maneira encontrada para melhorar a comunicação, segundo Miranda (2017) é a implantação de aplicativo para tablets e smartphones que dispõe de uma interface com o sistema INFOCRAS, permitindo que o usuário acompanhe em tempo real suas solicitações de agendamento. Os resultados dessa pesquisa, que contou com a parceria da Regulação com o setor de Tecnologia da Informação (TI), mostraram achados promissores, onde no primeiro mês após a divulgação do APP foi observado que a taxa de absenteísmo caiu de $40 \%$ para $4 \%$.

Claramente o fortalecimento dos centros reguladores contribui para o controle do absenteísmo, bem como das filas de espera. Diante da sua capacidade de intervir nos processos de prestação de serviços, pode-se afirmar que a regulação assistencial se configura como um importante instrumento para a melhoria no acesso, pois a partir do amplo conhecimento de suas potencialidades é possível traçar estratégias que promovam o acesso dos usuários ao SUS de forma equânime (Rocha, 2015).

Por outro lado, barreiras econômicas (custeio para alimentação e/ou transporte até o local da consulta/exame), bastante abordadas pelos estudos sobre o absenteísmo de pacientes, cuja realidade reflete como aspectos relacionados às políticas públicas podem contribuir para dificuldades no acesso aos serviços de saúde agravando, assim, as taxas de absenteísmo de usuários. Ainda assim, outros autores apontam a importância em se identificar as necessidades de atenção à saúde por meio da regulação médica e adoção de protocolos clínicos de regulação (Campoville, 2019; Siqueira, 2018; Rocha, 2015).

Cavalcanti et al. (2018) analisaram os desafios diretos e indiretos que podem ser enfrentados pela regulação, ou seja, considerando os níveis político e técnico. Para os autores, os desafios a nível político (indiretos) podem ser enfrentados conforme a regulação apresentar avanços consideráveis quanto os desafios a nível técnico (direto). Para tal, habilidades no planejamento e execução de um plano estratégico são fundamentais para subsidiar com maior precisão o nível político.

Diante do exposto, enfatiza-se que é fundamental existir uma agenda estratégica em que as gestões das regulações estejam atentas para agir intra e intersetorialmente, buscando pactuar ações que favoreçam uma maior eficiência e eficácia da oferta de procedimentos especializados. (Cavalcanti et al., 2018).

Portanto, considera-se que a integração entre Atenção Primária e especializada deve ser abordada como uma medida estratégica no enfrentamento de diversos desafios do setor de regulação, especialmente por parte de secretários de saúde e seu grupo de assessoramento direto, que estão na dimensão política (Cavalcanti et al., 2018).

\section{Conclusão}

Os debates acerca das perdas por absenteísmo de consultas e exames especializados, bem como as estratégias adotadas para reduzir essa problemática precisam ser ampliados para que essa questão seja abordada como possibilidade de 
estruturação do processo de trabalho das equipes, dos serviços, bem como da própria regulação em saúde na perspectiva de minimizar o absenteísmo, uma vez que suas causas são um quadro diversificado e complexo nos vários tipos de atenção.

Foi possível concluir que os estudos sobre essa temática chamam a atenção para a importância de envolver a rede de atenção como um todo para que medidas mais complexas e variadas, nos níveis estratégico, tático e operacional, com a implantação de estratégias tanto focais quanto sistêmicas, bem como a incorporação de sistemas de avaliação e monitoramento sejam buscadas para sanar a problemática do absenteísmo no acesso a atendimentos agendados.

Acredita-se que, provavelmente, a melhor estratégia para reduzir as perdas por absenteísmo de pacientes deve partir de uma abordagem estratégica mista, promovendo sistemas de lembretes de acordo com as necessidades da população envolvida, facilitando cancelamentos sempre que necessário, primando pela qualidade na comunicação, considerando os aspectos dos diferentes atores desse processo e não somente as perspectivas do sistema de saúde.

Em síntese, sinalizamos a importância em se desenvolver estudos posteriores sobre o tema em face da sua relevância para o bom funcionamento de uma rede de saúde integrada, os quais permitirão melhor detectar as fragilidades do sistema de saúde e, assim, suprimi-las e/ou mitiga-las.

\section{Referências}

Albieri, F. A. O., André, L. D. S., Malaquias, A. K., Moreira, R. A., Filipi Jr, J., \& Puccini, P. T. (2015). Agilidade no Acesso do Cidadão a Partir da Gestão da Fila de Espera da Atenção Especializada Ambulatorial em Busca da Melhoria da Eficácia da Rede de Atenção à Saúde. https://pesquisa.bvsalud.org/ses/resource/pt/sms-10224

Beltrame, S. M., Oliveira, A. E., Santos, M. A., \& SantosNeto, E. T. (2019). Non-attendance as a factor of waste: challenge for SUStainability in a universal health system. Saúde debate, 43(123):1015-30. https://doi.org/10.1590/0103-1104201912303

Bittar, O. J., Magalhães, A., Martines, C. M., Felizola, N. B., \& Falcão, L. H. (2016). Absenteísmo em atendimento ambulatorial de especialidades no estado de São Paulo. BEPA. Boletim epidemiológico paulista, 13(152):19-32. Disponível em: https://pesquisa.bvsalud.org/portal/resource/pt/ses-34318

Briatore, A., Tarsetti, E. V., Latorre, A., Quirós, F. G. B., Luna, D., Fuentes, N. A., Elizondo, C. M., Baum, A., Alonso Serena, M., Giunta, D. H. (2019). Causes of appointment attendance, nonattendance, and cancellation in outpatient consultations at a university hospital. International Journal of Health Planning and Management, 35(1):207-220. https://doi.org/10.1002/hpm.2890

Campoville, J. M. (2019). Estudo sobre absenteísmo em ambulatórios médicos Especializados no interior de São Paulo [Dissertação de Mestrado, Faculdade de Odontologia de Piracicaba da Universidade Estadual de Campinas]. http://repositorio.unicamp.br/jspui/bitstream/REPOS IP/335125/1/Campoville_JussaraMoraesHatae_M.pdf

Cavalcanti, R. P., Cavalcanti, J. C. M., Serrano, R. M. S. M., \&Santana, P. R. (2013). Absenteísmo de consultas especializadas nos sistemas de saúde público: relação entre causas e o processo de trabalho de equipes de saúde da família, João Pessoa - PB, Brasil. Revista Tempus, Actas de Saúde Coletiva, 18(2):63-84. https://doi.org/10.18569/tempus.v7i2.1344

Cavalcanti, R. P., Cruz, D. F., \& Padilha, W. W. (2018). Desafios da Regulação Assistencial na Organização do Sistema Único de Saúde. Revista Brasileira de Ciências da Saúde, 22(2):181-8. https://doi.org/10.4034/RBCS.2018.22.02.12

Costa, C. F. S., Duarte, P. M., \& Vaghetti, H. H. (2018). Absenteísmo em consultas especializadas referenciadas por unidade básica saúde da família: estudo de caso. Revista Saúde (Sta. Maria), 44(1):1-10. https://doi.org/10.5902/2236583420922

Cruz, D. F., Cavalcanti, R. P., Lucena, E. H., \& Padilha, W. W. (2018). A regulação da atenção à saúde bucal e o absenteísmo: cenários e possibilidades. REFACS, 6(2):228-237. http://seer.uftm.edu.br/revistaeletronica/index.php/refacs/article/view/2820.

Farias, C. M., Giovanella, L., Oliveira, A. E., \& Santos Neto, E. T. (2019). Tempo de espera e absenteísmo na atenção especializada: um desafio para os sistemas universais de saúde. Saúde debate, 43(5):190-204. https://doi.org/10.1590/0103-11042019S516

Farias, C. M., Moraes, L., Esposti, C. D., \& Santos Neto, E. T. (2020). Absenteísmo de usuários: barreiras e determinantes no acesso aos serviços de saúde. Revista Brasileira de Medicina de Família e Comunidade, 15(42):2239. https://doi.org/10.5712/rbmfc15(42)2239

Fenili, R., Silva, C. F., Silva, R. A., Taffarel, V. R. F., \& Correa, C. E. G. (2017, 4 a 6 de setembro). Avaliação da confirmação de agendamento prévio, como ferramenta de gestão para redução das taxas de absenteísmo. [Anais]. $1^{\circ}$ Congresso Internacional de Desempenho do Setor Público, Florianópolis, Brasil. http://cidesp.com.br/index.php/Icidesp/1 cidesp/paper/view/198/113

Foltz L. (2016). Enfrentamento do absenteísmo no centro de especialidades Médicas e odontológicas de Araucária/PR [Especialização em Gestão em Saúde da Universidade Federal do Paraná]. https://acervodigital.ufpr.br/bitstream/handle/1884/51693/R\%20-\%20E\%20-\%20LUCAS\%20FOLTZ.pdf?sequence= $1 \&$ isAllowed=y

Fonseca, J. J. S. (2002). Metodologia da pesquisa científica. UEC. 
Gurol-Urganci, I., Jongh, T., Vodopivec-Jamsek, V., Atun, R., \& Car, J. (2013). Mobile phone messaging reminders for attendance at healthcare appointments. Cochrane Library, 12(CD007458). https://doi.org/10.1002/14651858.CD007458.pub3

Hasvold, P. E., \& Wootton, R. (2011). Use of telephone and SMS reminders to improve attendance at hospital appointments: a systematic review. Journal of Telemedicine and Telecare, 17(7):358-64. https://doi.org/10.1258/jtt.2011.110707

Hernández García, I., Chaure Pardos, A., Moliner Lahoz, J., Prieto Andrés, P., Mareca Doñates, R., Giménez Júlvez, T., López Mendoza, H., García Montero, J. I., \& Aibar Remón, C. (2018). Absentismo, y factores asociados, en las citas programadas de una consulta externa de Medicina Preventiva. Journal of Healthcare Quality Research, 33(2):82-87. https://doi.org/10.1016/j.cali.2017.12.006

Jabalera Mesa, M. L., Asencio, J. M. M., Ruiz, F. R. (2015). Factores determinantes y coste económico del absentismo de pacientes en consultas externas de la agencia sanitaria costa del sol. Anales Sis San Navarra, 38(2):235-245. https://dx.doi.org/10.4321/S1137-66272015000200007

Jandrey, C. M., \& Drehmer, T. M. (2000). Absenteísmo no atendimento clínico-odontológico: o caso do Módulo de Serviço Comunitário (MSC) do Centro de Pesquisas em Odontologia Social (CPOS) - UFRGS. Revista da Faculdade de Odontologia de Porto Alegre, 40(2):24-8. https://lume.ufrgs.br/bitstream/handle/10183/23787/000269159.pdf?sequence=1\&isAllowed=y

Mazza, T.O., Ferreira, G. S., Picoli, R. M., \& Costa, A. L. (2019). Fatores do absenteísmo em primeira consulta num ambulatório de oncologia em um Hospital Universitário. Medicina (Ribeirão Preto, Online.), 52(1):24-33. http://dx.doi.org/10.11606/issn.2176-7262.v52i1p24-33

Mendes, K.D.S, Silveira, R.C.C.P., \& Galvão, C.M. (2008). Revisão integrativa: método de pesquisa para a incorporação de evidências na saúde e na enfermagem. Texto Contexto Enferm, 17(4): 758-64. https://www.scielo.br/pdf/tce/v17n4/18.pdf

Miranda, T. P. (2017). Implantação de aplicativo para acompanhamento de consultas e exames como estratégia para diminuir o absenteísmo dos usuários no Município de Caruaru-PE [Especialização em Saúde Pública da Escola de Saúde Pública de Pernambuco]. https://docs.bvsalud.org/biblioref/2020/10/1121412/tamine-poliane-da-mota-miranda_15305_assignsubmission_file_tam_dZlMq8G.pdf

Minayo, M. C. S. (1994). Ciência, técnica e arte: o desafio da pesquisa social. In: Maria Cecília de Souza Minayo (Org.). Pesquisa social: teoria, método e criatividade (pp. 9-29). Petrópolis: Vozes.

Murray, M., \& Tantau, C. (2000). Same-day appointments: exploding the access paradigm. Family Practice Management, 8:45-50. https://www.aafp.org/fpm/2000/0900/p45.html

Oleskovicz, M., Oliva, F. L., Grisi, C. C., Lima, A. C., \& Custódio, I. (2014). Técnica de overbooking no atendimento público ambulatorial em uma unidade do Sistema Único de Saúde. Cadernos de Saúde Pública, 30(5):1009-17. https://doi.org/10.1590/0102-311X00158612

Pires Filho, L. A. S., Azevedo Marques, J. M., Duarte, N. S. M., \& Moscovici L. (2019). Acesso Avançado em uma Unidade de Saúde da Família do interior do estado de São Paulo: um relato de experiência. Saúde debate, 43(121):605-13. https://doi.org/10.1590/0103-1104201912124

Rocha, A. P. (2015). Regulação Assistencial Ambulatorial no município do Rio de Janeiro - RJ: efeitos da inserção da APS na Regulação [Dissertação de Mestrado em Saúde Pública da Escola Nacional de Saúde Pública Sergio Arouca]. https://www.arca.fiocruz.br/bitstream/icict/14196/1/94.pdf

Rodrigues, G. R., Anhoque, C. F., Vasconcelos, K. A., Santos Neto, E. T., Sadovsky, A. D. I., \& Zandonade, E (2020). Impacto das mensagens de texto para redução do absenteísmo às consultas especializadas: um estudo aleatorizado. Revista cubana de información en ciencias de la salud, 31(3):e1566. https://pesquisa.bvsalud.org/portal/resource/pt/biblio-1138872

Silveira, G. S., Ferreira, P. R., Silveira, D. S., \& Siqueira, F. C. V. (2018). Prevalência de absenteísmo em consultas médicas em unidade básica de saúde do sul do Brasil. Revista Brasileira de Medicina de Família e Comunidade, 13(40):1-7. http://dx.doi.org/10.5712/rbmfc13(40)1836

Siqueira, U. B. (2018). Análise do impacto da fila de espera na probabilidade de absenteísmo em exames e consultas [Monografia apresentada ao Curso de Saúde Coletiva da Universidade Federal de Pernambuco, Centro Acadêmico de Vitória. https://repositorio.ufpe.br/bits tream/123456789/26021/1/SIQUEIRA\%2C\%20\%C3\%9Arsula\%20Beatriz\%20Galv\%C3\%A3o.pdf

Souza RR. (2002). Dados Gerais. O SUS. In: Souza RR. O sistema Público de Saúde Brasileiro. Brasília: Editora MS; 2002. Segunda Parte: O Histórico do Sistema de Saúde Brasileiro, 16-17.

Torezani, G. S. (2018). Estudo sobre as Faltas às consultas e seus motivos: uma análise da comunidade Sete Anões - Mesquita/RJ [Especialização em Saúde da Família da Universidade Aberta do SUS]. https://ares.unasus.gov.br/acervo/handle/ARES/7960

Zirkle, M. S., \& McNelles, L. R. (2011). Nonattendance at a hospital-based otolaryngology clinic: a preliminar analysis within a universal healthcare system. Ear, Nose \& Throat Journal, 90(8):E32-4. https://pubmed.ncbi.nlm.nih.gov/21853431/ 